\title{
Shikonin induces an anti-tumor effect on murine mammary cancer via p38-dependent apoptosis
}

\author{
JIUXIANG XU ${ }^{1,2^{*}}$, KEIICHI KOIZUMI ${ }^{2 *}$, MENGXIN LIU $^{2}$, YUSUKE MIZUNO ${ }^{2}$, MIKIKO SUZAKI $^{2}$, \\ HIROFUMI IITSUKA ${ }^{2}$, AKIKO INUJIMA ${ }^{2}$, MAKOTO FUJIMOTO ${ }^{1}$, \\ NAOTOSHI SHIBAHARA ${ }^{2}$ and YUTAKA SHIMADA ${ }^{1}$ \\ ${ }^{1}$ Department of Japanese Oriental Medicine, Graduate School of Medicine and Pharmaceutical Sciences; \\ ${ }^{2}$ Division of Kampo Diagnostics, Institute of Natural Medicine, University of Toyama, Toyama 930-0194, Japan
}

Received November 5, 2017; Accepted November 15, 2018

DOI: $10.3892 /$ or.2019.6966

\begin{abstract}
Breast cancer is the most common malignancy in women. Apoptosis is important for tumor suppression and may delay cancer progression. It was found that shikonin induced apoptosis in 4T1 murine mammary cancer cells and MDA-MB-231 human breast cancer cells in vitro. Total p38 and c-Jun N-terminal kinase (JNK) levels were maintained in 4T1 cells, and p38 phosphorylation, but not JNK phosphorylation, was significantly increased. Caspase-3/7 activity was detected, which suggested that the p38 pathway, but not the JNK signaling pathway, induced apoptosis in 4T1 cells. The anti-tumor effects of shikonin on orthotopic mouse models were also examined. On day 7 after inoculation of 4T1 cells into mice, tumor volumes in the shikonin-treated and the control groups began to differ. On day 13, tumors were weighed, and shikonin was revealed to suppress tumor growth in the orthotopic 4T1 model in vivo. In conclusion, shikonin is a potential anti-tumor drug for breast cancer.
\end{abstract}

\section{Introduction}

Breast cancer is the most common malignancy among women (1). Most breast cancer-associated mortalities result from tumor metastasis, which frequently follows a specific dissemination pattern. Breast cancer is a complex molecular disease in which gene mutations result in uncontrolled cell growth and proliferation. These mechanisms mainly involve cell growth and cell death pathways. As apoptosis has an important role in tumor surveillance (2), the induction of apoptosis may result in tumor suppression. Recently, there has been

Correspondence to: Professor Keiichi Koizumi, Division of Kampo Diagnostics, Institute of Natural Medicine, University of Toyama, 2630 Sugitani, Toyama 930-0194, Japan

E-mail: kkoizumi@inm.u-toyama.ac.jp

${ }^{*}$ Contributed equally

Key words: shikonin, 4T1, murine mammary cancer, p38 signaling pathway, apoptosis renewed interest in finding natural therapeutic compounds that can induce apoptosis and inhibit cell proliferation to treat cancer (3-5).

Shikonin is a natural naphthoquinone derivative isolated from the traditional medical herb Lithospermum erythrorhizon. Shikonin has anti-tumor, anti-inflammatory (6), anti-microbial (7), and anti-thrombotic properties (8). Prolonged exposure of shikonin to cancer cells does not result in chemoresistance, and shikonin inhibits the expression of inflammatory cytokines and associated signaling pathways (9). Shikonin decreases the expression of tumor necrosis factor (TNF) $\alpha$, interleukin (IL)12, IL6, IL1 $\beta$, IL2, and interferon (IFN) $\gamma$ (10), inhibits extracellular signal-regulated kinase (ERK) $1 / 2$ and c-Jun N-terminal kinase (JNK) signaling (11), and reduces the expression of nuclear factor $(\mathrm{NF})-\kappa \mathrm{B}$ and signal transducer and activator of transcription 3 (STAT3) transcription factors. Shikonin inhibits the proteasome and modulates cancer cell metabolism by inhibiting the tumor-specific pyruvate kinase M214-16. Shikonin causes cell cycle arrest and induces necroptosis in various cancer types. In addition, shikonin inhibits matrix metalloproteinase (MMP) 9 and integrin $\beta 1$ expression, and decreases the invasive potential of cancer cells. The p38 and JNK signaling pathways are targeted by anti-cancer agents to induce apoptosis in murine 4T1 cells $(12,13)$. Collectively, shikonin modulates various signaling pathways and elicits responses in various cancer types.

In breast cancer, shikonin induces cell death and inhibits migration $(14,15)$, but the mechanisms are not yet well understood. The importance of signaling pathways modulated by shikonin in cancerous and non-cancerous models has previously been examined with respect to breast carcinoma growth, metastasis and tumorigenicity.

In the present study, the molecular mechanisms underlying the effects of shikonin on the inhibition of $4 \mathrm{~T} 1$ cell proliferation and the induction of apoptosis were investigated in vitro and in vivo.

\section{Materials and methods}

Compounds and reagents. Shikonin was purchased from Sigma-Aldrich (Merck KGaA, Darmstadt, Germany) 
and dissolved in dimethyl sulfoxide (DMSO); the final concentration was $100 \mathrm{mM}$. SB203580 and SP600125 inhibitors were purchased from Wako Pure Chemical Industries, Ltd. (Osaka, Japan). A Cell Counting Kit-8 (CCK8) was purchased from Dojindo Molecular Technologies, Inc. (Kumamoto, Japan). A Caspase-3/7 Fluorescence Assay kit was purchased from Cayman Chemical Company (Ann Arbor, MI, USA). Antibodies for total JNK, phospho (p)-JNK, total p38, p-p38, and $\beta$-actin were purchased from Cell Signaling Technology, Inc. (Danvers, MA, USA). Caspase-3/7 inhibitor (N-Ac-DEVD-CHO) was purchased from Cayman Chemical Company. Medetomidine, midazolam, and butorphanol were purchased from Nippon Zenyaku Kogyo Co., Ltd. (Fukushima, Japan), Astellas Pharma Inc (Tokyo, Japan) and Meiji Seika Pharma Co., Ltd. (Tokyo, Japan), respectively.

Cell lines and cell culture conditions. The 4T1 murine mammary cancer and MDA-MB-231 human breast cancer cells (ATCC, Manassas, VA, USA) were cultured in RPMI-1640 medium supplemented with $10 \%$ (v/v) fetal bovine serum (FBS) (both from Thermo Fisher Scientific, Inc., Waltham, MA, USA) and $1 \%$ penicillin and streptomycin at $37^{\circ} \mathrm{C}$ in a humidified atmosphere containing $5 \% \mathrm{CO}_{2}$. All assays were performed when the cells reached $70 \%$ confluency.

Mice and the orthotopic model of murine mammary cancer cells. Female BALB/c mice (6 weeks old) were purchased from Sankyo Labo Service Corporation, Inc. (Tokyo, Japan). This study was carried out in strict accordance with the Guide for the Care and Use of Laboratory Animals of University of Toyama (Toyama, Japan). The protocol was approved by the Committee on the Ethics of Animal Experiments of University of Toyama. All surgery was performed under optimal anesthesia [a combination anesthetic with medetomidine $(0.3 \mathrm{mg} / \mathrm{kg})$, midazolam $(4.0 \mathrm{mg} / \mathrm{kg})$ and butorphanol $(5.0 \mathrm{mg} / \mathrm{kg})]$, and all efforts were made to minimize suffering.

The animals were grouped according to body weight, and housed under controlled laboratory conditions, including a $12 \mathrm{~h}$ light/12 $\mathrm{h}$ dark cycle and free access to standard diet and water. For cancer induction, the mice received a single subcutaneous injection of $1 \times 10^{5} 4 \mathrm{~T} 1$ breast cancer cells suspended in phosphate-buffered saline (PBS) in the fourth mammary fat pad. Tumors were inspected and measured every other day: Tumor volume $\left(\mathrm{mm}^{3}\right)=1 / 2$ [length $(\mathrm{mm}) \mathrm{x}$ width $\left.{ }^{2}\left(\mathrm{~mm}^{2}\right)\right]$.

Cell proliferation assay. The 4T1 cells were cultured into 6-cm dishes with RPMI-1640 medium, supplemented with $10 \%(\mathrm{v} / \mathrm{v}) \mathrm{FBS}$ and $1 \%$ penicillin/streptomycin at $37^{\circ} \mathrm{C}$ in a humidified atmosphere containing $5 \% \mathrm{CO}_{2}$. After a $24 \mathrm{~h}$ incubation period, adherent cells covered $70 \%$ of the dish surface. Cells were stimulated with different concentrations of shikonin (0-8 $\mu \mathrm{M})$ for $24 \mathrm{~h}$ and then imaged with a Type 1109137001 light microscope (Leica, Wetzlar, Germany).

Cytotoxicity assay. Cytotoxicity in 4T1 and MDA-MB-231 cells was measured with a CCK-8 assay, following the manufacturer's instructions. Briefly, cells were seeded at a density of $1 \times 10^{5} / 100 \mu \mathrm{l} /$ well in 96 -well plates, and then incubated at $37^{\circ} \mathrm{C}$ in a humidified atmosphere containing $5 \% \mathrm{CO}_{2}$ for $12 \mathrm{~h}$ in RPMI-1640 medium. Cells were treated with different<smiles>CC(C)=CCC(O)C1=CC(=O)c2c(O)ccc(O)c2C1=O</smiles>

Figure 1. Structure of shikonin.

concentrations of shikonin $(0-4.0 \mu \mathrm{M})$. After a 24-h incubation period, the wells were washed twice with cold PBS (-) (high temperature sterilized) and then $100 \mu \mathrm{l}$ RPMI-1640 medium and $10 \mu$ CCK- 8 were added to each well; the cells were incubated for another $2 \mathrm{~h}$. Optical density was detected at $450 \mathrm{~nm}$ with a Filter Max F5 Multi-Mode microplate reader (Molecular Devices, San Jose, CA, USA).

Caspase-3/7 fluorescence assay and inhibitor experiment. To examine apoptosis in 4T1 and MDA-MB-231 cells after shikonin treatment, a caspase-3/7 fluorescence assay was used. Cells were seeded in a 96-well plate at a density of $1 \times 10^{4} /$ well in RPMI-1640 medium at $37^{\circ} \mathrm{C}$ in a humidified atmosphere containing $5 \% \mathrm{CO}_{2}$ overnight. The cells were treated with shikonin for $12 \mathrm{~h}$ in an incubator at $37^{\circ} \mathrm{C}$ and $5 \% \mathrm{CO}_{2}$. The plate was centrifuged at $400 \mathrm{x} \mathrm{g}$ for $5 \mathrm{~min}$. The culture medium was aspirated, $200 \mu \mathrm{l}$ assay buffer was added to each well, the plate was centrifuged at $630 \mathrm{x} \mathrm{g}$ for $5 \mathrm{~min}$, after which the supernatant was aspirated. Subsequently, $50 \mu 1$ cell-based assay lysis buffer was added to each well. The cells were incubated with gentle shaking in an orbital shaker for $30 \mathrm{~min}$ at room temperature and then the plate was centrifuged at $630 \mathrm{x} \mathrm{g}$ for $10 \mathrm{~min}$. Following this, $45 \mu \mathrm{l}$ supernatant was transferred from each well to a corresponding well in a new black microtiter 96-well plate. Afterward, $5 \mu 1$ assay buffer was added to the wells for sample activity measurement, and $5 \mu \mathrm{l}$ caspase-3/7 inhibitor solution was used to test assay specificity. Then, $50 \mu \mathrm{l}$ caspase- $3 / 7$ substrate solution was added to each well and the plate was incubated at $37^{\circ} \mathrm{C}$ for $30 \mathrm{~min}$. The fluorescence intensity of each well was measured at an excitation wavelength of $485 \mathrm{~nm}$ and an emission wavelength of $535 \mathrm{~nm}$. The 4T1 cells were pretreated with inhibitors of p38 (SB203850, $10 \mu \mathrm{M}$ ) and JNK (SP600125, $10 \mu \mathrm{M}$ ) for $15 \mathrm{~min}$ in a $37^{\circ} \mathrm{C}$ incubator containing $5 \% \mathrm{CO}_{2}$. Cells were washed with cold PBS (-) twice after pretreatment, and then treated with $2 \mu \mathrm{M}$ shikonin for $12 \mathrm{~h}$ in an incubator. Afterward, the same protocol was followed, but the caspase-3/7 inhibitor was removed. Instead, assay buffer was added to all wells after the supernatant was transferred to the corresponding well in a new black microtiter 96-well plate.

Western blotting assay. 4T1 cells were categorized into two groups: The control group, which had 4T1 cells with/without $2 \mu \mathrm{M}$ shikonin treatment for $24 \mathrm{~h}$, and the treatment group, which had 4T1 cells treated with $2 \mu \mathrm{M}$ shikonin for $24 \mathrm{~h}$ and pretreated with/without p38 and JNK inhibitors for $15 \mathrm{~min}$. Whole cell lysates were prepared with lysis buffer (20 mM HEPES-NaOH, 0.3 M NaCl, $1.5 \mathrm{mM} \mathrm{MgCl}_{2}$, $0.2 \mathrm{mM}$ EDTA, $0.001 \%$ Triton X-100, $1 \mathrm{mM}$ DTT, $1 \mathrm{mM}$ sodium orthovanadate, $20 \mathrm{mM} \beta$-glycerophosphate 
A
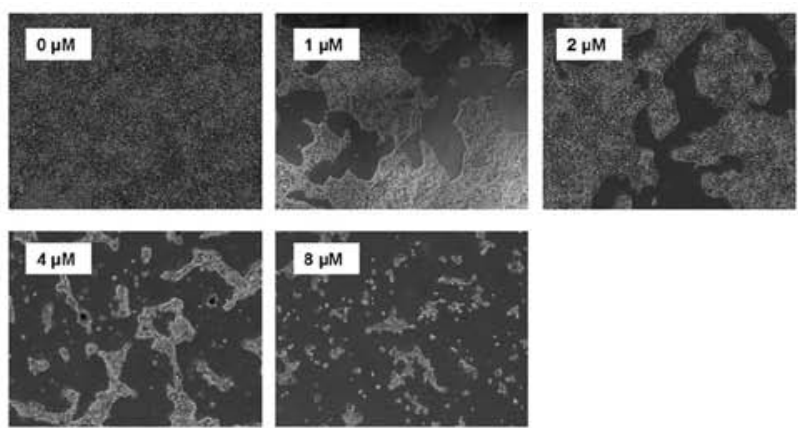

C
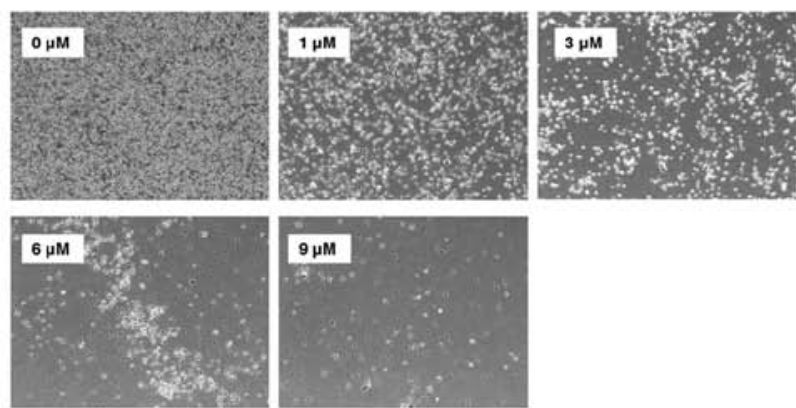
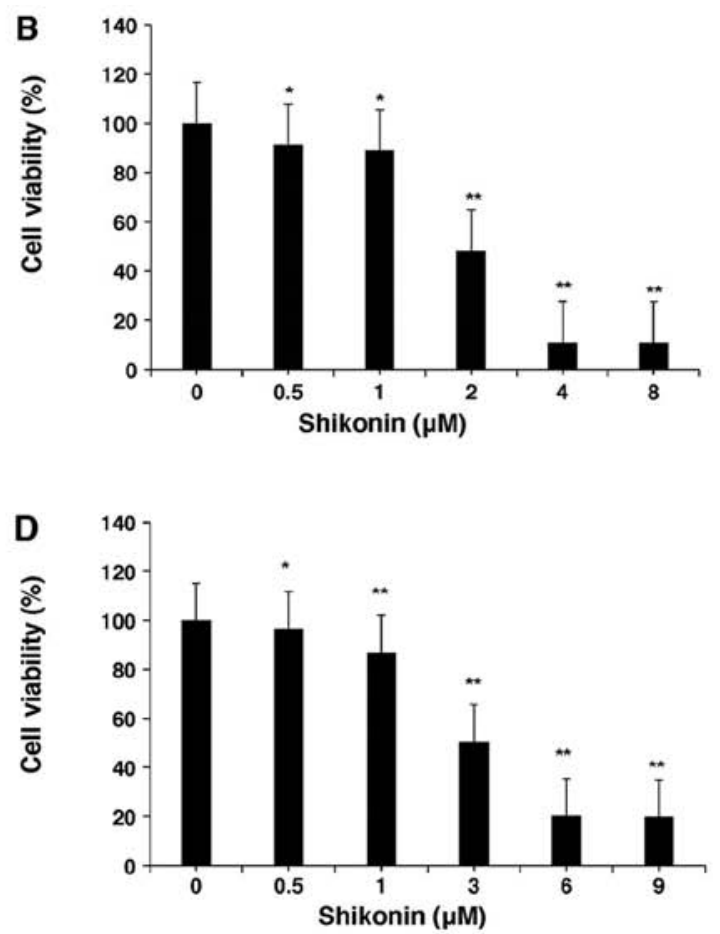

Figure 2. Shikonin induced 4T1 and MDA-MB-231 cell death in a dose-dependent manner. (A and B) 4T1 and MDA-MB-231 cells were treated with increasing concentrations of shikonin $(0-9 \mu \mathrm{M})$ for $24 \mathrm{~h}$, as indicated. Inhibition of (C) 4T1 and (D) MDA-MB-231 cell proliferation by shikonin. Data are presented as the means \pm standard deviation $(\mathrm{n}=3) ;{ }^{*} \mathrm{P}<0.05,{ }^{* *} \mathrm{P}<0.01$, compared with the control $(0 \mu \mathrm{M}$ shikonin $)$.

disodium salt hydrate, $10 \mu \mathrm{g} / \mathrm{ml}$ aprotinin, $10 \mu \mathrm{g} / \mathrm{ml}$ leupeptin, and $1 \mathrm{mM}$ PMSF). Protein concentrations in lysates were quantified by a protein assay (Bio-Rad Laboratories, Inc., Hercules, CA, USA). Total protein was resolved via $12 \%$ SDS-PAGE, and then transferred to a PVDF membrane at $20 \mathrm{~mA}$ for $30 \mathrm{~min}$. The membranes were blocked with non-fat milk for one night at $4^{\circ} \mathrm{C}$. Primary antibodies $[\beta$-actin (13E5) rabbit mAb, no. 4970; p38 MAPK antibody, no. 9212; p-p38 MAPK (Thr180/Tyr182) antibody, no. 9211; SAPK/JNK antibody, no. 9252; p-SAPK/JNK (Thr183/Tyr185) antibody, no. 9251; all from Cell Signaling Technology, Danvers, MA, USA] were diluted 1:1,000, and incubated for $90 \mathrm{~min}$ at room temperature. After washing with Tris-buffered saline and Tween 20 three times (10 min each), the secondary antibody (diluted 1:2,000) was added to the membrane and incubated at room temperature for $45 \mathrm{~min}$. $\beta$-actin was used as the loading control and for normalization. The density of the blots was quantified using Image Studio Lite v.5.2 software (LI-COR Biosciences, Lincoln, NE, USA).

Statistical analysis. All data are expressed as the means \pm standard deviation from three independent experiments. Image Studio Lite v.5.2 software was used for analysis. Differences between two groups were determined using a Student's t-test and Bonferroni's correction, comparing the treated groups with the control. $\mathrm{P}<0.05$ was considered to indicate a statistically significant difference.

\section{Results}

Cell proliferation. 4T1 and MDA-MB-231 cells were used to investigate the effect of shikonin on proliferation (Fig. 1).
As presented in Fig. 2A, a confluent monolayer of 4T1 cells was observed without shikonin treatment, but confluence was reduced with increasing concentrations of shikonin $(0-8 \mu \mathrm{M})$. Shikonin significantly inhibited $4 \mathrm{~T} 1$ cell proliferation in a dose-dependent manner compared with that of the control, a cytotoxic effect that was more evident after $24 \mathrm{~h}$. The dose of shikonin that effectively inhibited $50 \%$ of the growth of $4 \mathrm{~T} 1$ cells after $24 \mathrm{~h}$ was $2 \mu \mathrm{M}$ (Fig. $2 \mathrm{~B}$ ); additionally, the IC50 of MDA-MB-231 cells was $3 \mu \mathrm{M}$ (Fig. 2C and D)

Involvement of caspase-3/7 in shikonin-induced apoptosis. Treatment of 4T1 cells with 2 and $4 \mu \mathrm{M}$ (Fig. 3A) shikonin resulted in asignificantincrease in caspase-3/7 activity compared with the control. Shikonin also increased caspase-3/7 activity in MDA-MB-231 cells (Fig. 3B). The involvement of caspase in shikonin-induced apoptosis was examined by pretreating cells with SB203580 (p38 inhibitor, $10 \mu \mathrm{M}$ ) (Fig. 3C) or SP600125 (JNK inhibitor, $10 \mu \mathrm{M}$ ) (Fig. 3D). SB203580, but not SP600125, significantly inhibited shikonin-induced apoptosis in 4T1 cells by decreasing caspase activity. These results suggested that shikonin-induced apoptosis in breast cancer cells is caspase-dependent and regulated by the p38 signaling pathway.

Involvement of the p38 and JNK pathways in shikonin-induced apoptosis. Mitogen-activated protein kinases, such as JNK and p38, control cell proliferation and death (16), and shikonin can regulate JNK and p38 activation in prostate cancer. To determine the effect of shikonin in 4T1 cells, the activation of p38 and JNK signaling pathways was investigated via western blotting. Total p38 and JNK levels were similar when compared with those in the non-treated group (Fig. 4A). As presented 

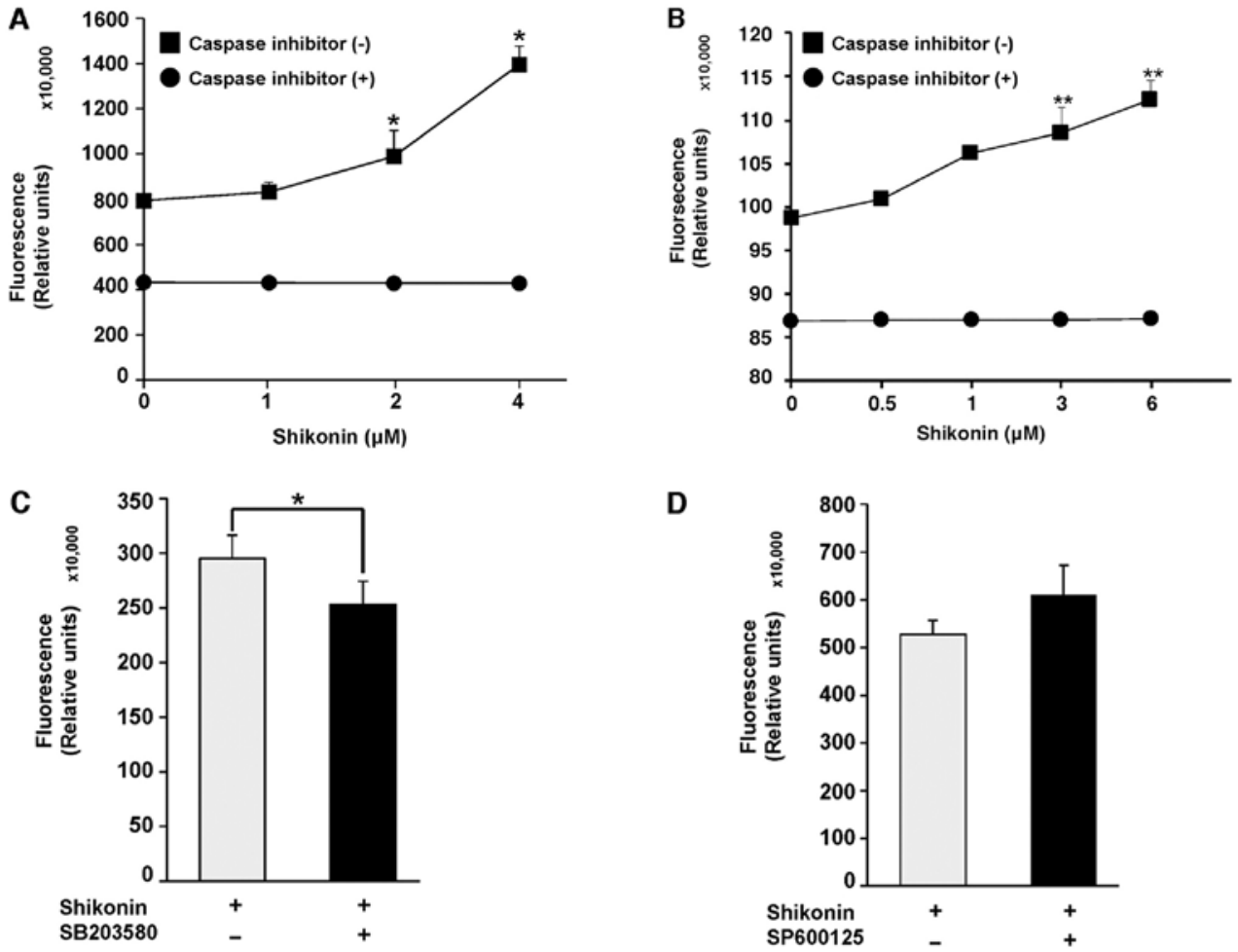

Figure 3. Activation of caspase-3/7 in $4 \mathrm{~T} 1$ cells. (A) $4 \mathrm{~T} 1$ cells were treated with shikonin $(0-4 \mu \mathrm{M})$ and (B) MDA-MB-231 cells were treated with shikonin $(0-6 \mu \mathrm{M})$, with/without a caspase-3/7 inhibitor (N-Ac-DEVD-CHO). Caspase-3/7 activity increased after shikonin treatment. Data are presented as the means \pm standard deviation ( $\mathrm{n}=3$ ); $\mathrm{P}<0.05,{ }^{* *} \mathrm{P}<0.01$, compared with the control (treated with caspase-3/7 inhibitor). (C) $4 \mathrm{~T} 1$ cells were divided into two groups: Treated with $2 \mu \mathrm{M}$ shikonin, with/without SB203580 pretreatment. (D) 4T1 cells were divided into two groups: Treated with $2 \mu \mathrm{M}$ shikonin, with/without SP600125 pretreatment. Data are presented as the means \pm standard deviation $(\mathrm{n}=3)$; ${ }^{*} \mathrm{P}<0.05$, compared with the control [caspase inhibitor $(+)$ ].
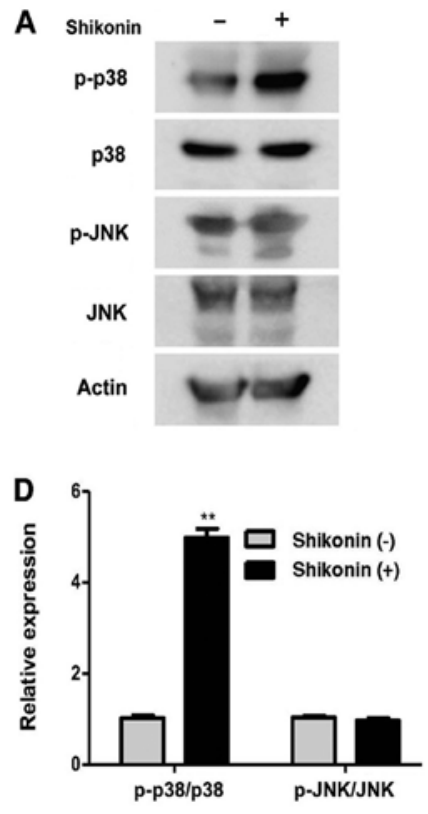

B

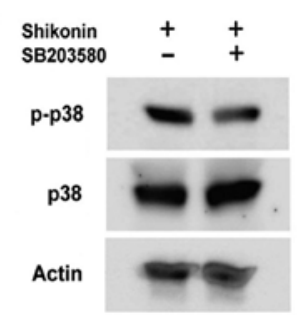

E

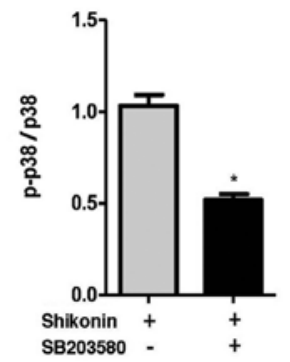

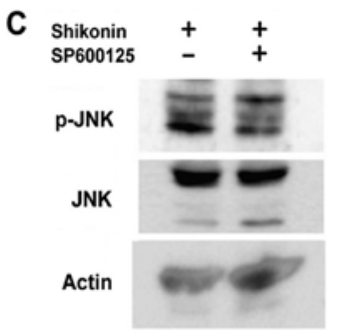

$\mathbf{F}$

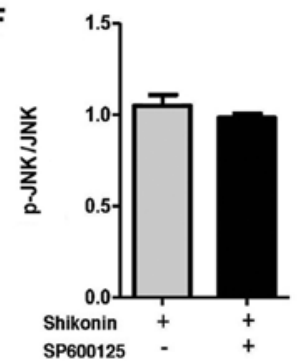

Figure 4. Western blot analysis of the inhibitory effect of shikonin on 4T1 cells. Cells were categorized into two groups: The control group, 4T1 cells not treated with $2 \mu \mathrm{M}$ shikonin for $24 \mathrm{~h}$; and the treatment group, 4T1 cells pretreated with/without $\mathrm{p} 38$ and JNK inhibitors for 15 min and then treated with $2 \mu \mathrm{M}$ shikonin for $24 \mathrm{~h}$. (A) Phosphorylation of $\mathrm{p} 38$, but not of JNK, was increased by $2 \mu \mathrm{M}$ shikonin. (B) The expression levels of p-p38 were suppressed by SB203580. (C) The expression of p-JNK also slightly decreased with SP600125 treatment. (D) The relative expression levels of (A). (E) The relative expression levels of (B). (F) The relative expression levels of (C). Data are presented as the means \pm standard deviation $(\mathrm{n}=3)$; ${ }^{*} \mathrm{P}<0.05$, ${ }^{* *} \mathrm{P}<0.01$, compared with the control (0 $\mu \mathrm{M}$ shikonin). JNK, c-Jun N-terminal kinases; p-, phosphorylated.

in Fig. 4B and $\mathrm{C}$, both groups of $4 \mathrm{~T} 1$ cells were treated with $2 \mu \mathrm{M}$ shikonin for $24 \mathrm{~h}$. But p38 phosphorylation increased with shikonin treatment (Fig. 4D) and p-JNK levels did not change (Fig. 4D). In the group pretreated with SB203580, p-p38 

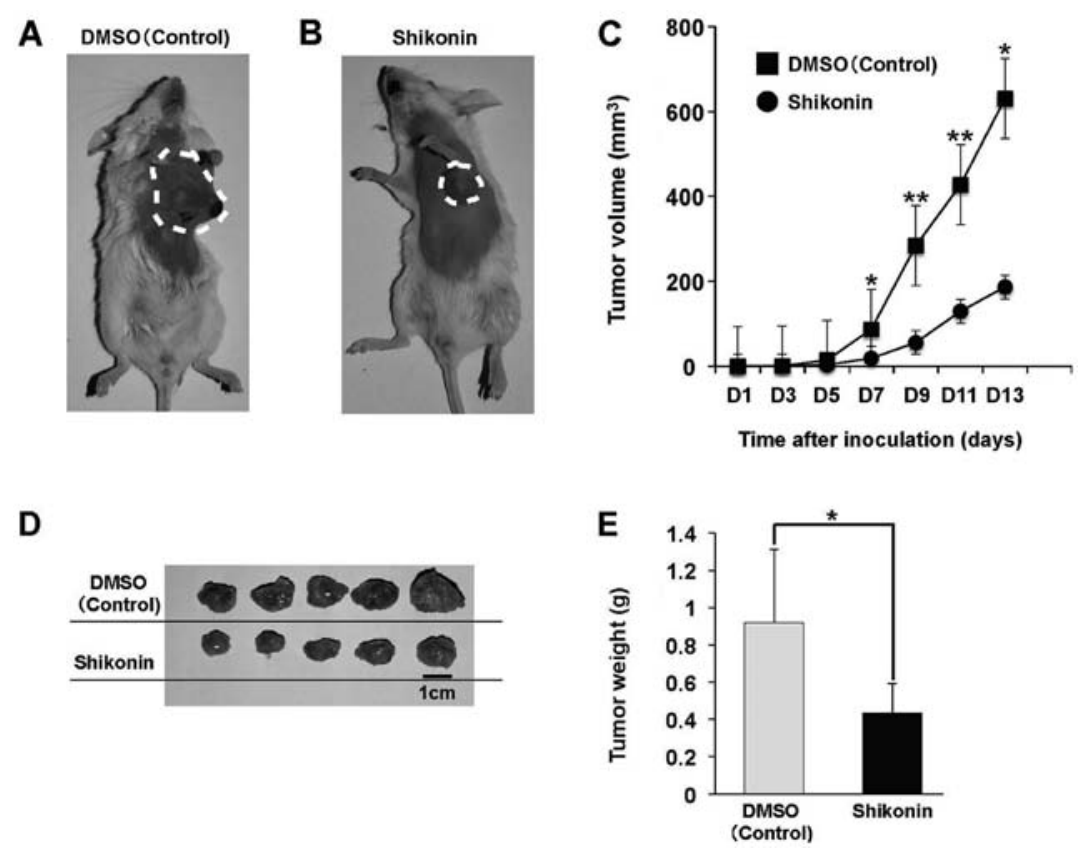

Figure 5. Orthotopic transplantation of 4T1 mammary tumor cells. (A) Representative example of a DMSO-treated mouse. (B) Representative example of a shikonin-treated mouse. (C) Mice were injected with $1 \times 10^{5} 4 \mathrm{~T} 1$ cells, and tumor volumes were monitored between days 1 and 13 . (D) Final tumor weight in the DMSO group (upper) and the shikonin group (lower). (E) Tumor average weight. Data are presented as the means \pm standard deviation $(\mathrm{n}=5) ;{ }^{*} \mathrm{P}<0.05,{ }^{* * *} \mathrm{P}<0.01$, compared with the control (DMSO group). DMSO, dimethyl sulfoxide

levels were markedly suppressed (Fig. 4B and E). However, pretreatment with SP600125 only slightly decreased p-JNK levels (Fig. 4C and F). Thus, the p38 signaling pathway is associated with shikonin-induced apoptosis. Statistical analysis through Image Studio Lite v.5.2 software from LI-COR and showed a significant increase in the expression of p-p38 in groups where shikonin was administered, and decrease in the expression of p-p38 in groups which pretreated with SB203580.

Anti-tumor effect of shikonin on 4T1 cancer. To determine whether shikonin inhibits tumor growth in vivo, an orthotopic 4T1 model was used. A single subcutaneous injection of $1 \times 10^{5} 4 \mathrm{~T} 1$ breast cancer cells suspended in PBS was administered into the fourth mammary fat pad in mice. Shikonin $(2 \mathrm{mg} / \mathrm{kg})$ or $1 \%$ DMSO was injected on the same day as $4 \mathrm{~T} 1$ cell implantation. Tumor volume was measured with a Vernier caliper every other day at the same time as shikonin or DMSO injection. Shikonin suppressed orthotopic 4T1 tumor growth in vivo (Fig. 5). From day 7, tumor volumes started to differ between the treatment and DMSO groups, and there were significant differences up to day 13 (Fig. 5A). On day 13, mice were sacrificed, and tumor sizes were measured (Fig. 5B).

The humane endpoints of the study (tumors did not exceed $20 \mathrm{~mm}$ in diameter; feeding, drinking and walking behaviors were not observed) were set in accordance with the Guide for the Care and Use of Laboratory Animals of University of Toyama. Tumor weights revealed the anti-tumor effect of shikonin on $4 \mathrm{~T} 1$ breast cancer (Fig. 5C).

\section{Discussion}

It has been found that shikonin exhibits anti-proliferation and apoptosis-inducing effects on 4T1 murine mammary cancer and MDA-MB-231 human breast cancer cells
(Figs. 1-3) (17,18). Various medicinal plants have been examined for anti-tumor compounds (19). Here, it was found that shikonin triggers apoptosis and diminishes the growth, size and weight of tumors in an orthotopic BALB/c mouse model. Inhibition of 4T1 tumor growth has also been observed in vivo $(20,21)$. Scrophularia oxysepala extract decreases the growth of breast cancer cells and reduces tumor mass without significantly affecting body weight, which suggests that the plant is not toxic (22). It was found that tumor volume and size were significantly greater in the control (DMSO) group than in the shikonin-treated group (Fig. 5), which suggests that shikonin induces apoptosis in tumor cells. Thus, shikonin may be an ideal therapeutic tool for breast cancer.

Reactive oxygen species are the most important mediators of shikonin-induced apoptosis in Bcr/Abl-positive chronic leukemia cells through JNK activation. Shikonin also inhibits Akt and receptor-interacting protein/NF- $\mathrm{KB}$ pathways in hepatocellular carcinoma cells $(23,24)$. However, the mechanism of shikonin-induced apoptosis in various cancer cells is unclear. Shikonin pretreatment attenuates ConA-induced acute liver injury by inhibiting apoptosis and autophagy through suppression of the JNK pathway (25). Apoptosis, the cell cycle, and migration are closely associated with the p38 pathway (26). The p38 signaling pathway is also involved in pulmonary fibrosis, and p38 expression significantly increases in lung homogenates from patients with idiopathic pulmonary fibrosis in epithelial, endothelial, fibroblasts and smooth muscle cells $(27,28)$.

The $\mathrm{p} 38$ and JNK pathways were investigated to understand how shikonin induces 4T1 tumor apoptosis (Fig. 4A and D). Apoptosis limits cancer propagation, and affects the development and progression. Apoptosis is modulated by many anti-apoptotic and pro-apoptotic factors. JNK and p38 are critical for 4T1 cell apoptosis $(29,30)$. 4T1 cells were pretreated 
with p38 and JNK inhibitors (SB203580 and SP600125, respectively) for 15 min before cells were incubated with shikonin, and several proteins were examined, including $\mathrm{p} 38$, p-p38, JNK and p-JNK. Shikonin-treated cells increased p-p38 expression while maintaining the same level of p38 and JNK expression. After the $\mathrm{p} 38$ signaling pathway was blocked with SB203580, the expression of p-p38 decreased compared with that of cells not pretreated with inhibitor (Fig. 4B and E). The expression of p-JNK was not changed by pretreatment with SP600125 (Fig. 4C and F). Thus, the p38 signaling pathway is a major regulator of shikonin-induced apoptosis in 4T1 cells.

Caspase-3/7 activation is responsible for the final step of apoptosis $(31,32)$ and reflects the degree of apoptosis in various cancer cells (33). To determine whether the anti-proliferative effect of shikonin was associated with the induction of apoptosis, a fluorescence quantification assay was used. Treatment of $4 \mathrm{~T} 1$ cells with $2 \mu \mathrm{M}$ shikonin significantly increased caspase-3/7 activity (Fig. 3A), which suggests that shikonin inhibits 4T1 cell proliferation and induces apoptosis by activating caspase-3/7. In addition, SB203580 reduced shikonin-induced 4T1 cell apoptosis by decreasing caspase-3/7 activity (Fig. 3C), though SP600125 did not have a significant effect (Fig. 3C). In the present study, shikonin exerted an anti-tumor effect by inhibiting proliferation and inducing 4T1 and MDA-MB-231 cell apoptosis. Shikonin was previously found to decrease the migration (34) and invasion (15) ability of MCF-7 and MDA-MB-231 human breast cancer cells. Therefore, the results suggest that shikonin can inhibit human breast cancer metastasis. The effect of shikonin on 4T1 cancer cells will be investigated in a future study. Further research on the anti-tumor mechanisms of shikonin is needed to determine whether there are other ligands involved in immunosuppression, the immune response, and the delay of disease progression.

In conclusion, shikonin induced p38-dependent apoptosis in 4T1 and MDA-MB-231 cells and markedly suppressed in vitro proliferation. Furthermore, shikonin delayed 4T1 cell growth in an orthotopic mouse model. Thus, shikonin may be a candidate drug to treat breast cancer.

\section{Acknowledgements}

The authors thank Yoshiki Takeshita and Takeshi Eto for providing technical support.

\section{Funding}

This research was partially supported by a Grant-in-Aid for the Cooperative Research Project from the Institute of Natural Medicine, University of Toyama in 2014, 2015 and 2017 (grant nos. 2014S, 2015S and 2017A1).

\section{Availability of data and materials}

Requests for materials should be addressed to KK (kkoizumi@ inm.u-toyama.ac.jp).

\section{Authors' contributions}

JX and KK designed the research; JX, ML, YM, MS, HI, AI, MF, NS and YS performed the experiments and prepared all the figures. JX, KK, NS and YS wrote the paper. All authors discussed and agreed on the results, read and approved the final manuscript.

\section{Ethics approval and consent to participate}

The protocol was approved by the Committee on the Ethics of Animal Experiments of University of Toyama (Toyama, Japan).

\section{Patient consent for publication}

Not applicable.

\section{Competing interests}

The authors declare no competing financial interests.

\section{References}

1. Jemal A, Bray F, Center MM, Ferlay J, Ward E and Forman D: Global cancer statistics. CA Cancer J Clin 61: 69-90, 2011.

2. Wakimoto R, Ono M, Takeshima M, Higuchi T and Nakano S: Differential anticancer activity of pterostilbene against three subtypes of human breast cancer cells. Anticancer Res 37: 6153-6159, 2017.

3. Chavoshi H, Vahedian V, Saghaei S, Pirouzpanah MB, Raeisi M and Samadi N: Adjuvant therapy with silibinin improves the efficacy of paclitaxel and cisplatin in MCF-7 breast cancer cells. Asian Pac J Cancer Prev 18: 2243-2247, 2017.

4. Zhao Y, Tian B, Wang Y and Ding H: Kaempferol sensitizes human ovarian cancer cells-OVCAR-3 and SKOV-3 to tumor necrosis factor-related apoptosis-inducing ligand (TRAIL)-induced apoptosis via JNK/ERK-CHOP pathway and up-regulation of death receptors 4 and 5. Med Sci Monit 23: 5096-5105, 2017.

5. Wu WS, Chien CC, Liu KH, Chen YC and Chiu WT: Evodiamine prevents glioma growth, induces glioblastoma cell apoptosis and cell cycle arrest through JNK activation. Am J Chin Med 45: 879-899, 2017.

6. Bi Y, Zhu Y, Zhang M, Zhang K, Hua X, Fang Z, Zhou J, Dai W, Cui Y, Li J, et al: Effect of shikonin on spinal cord injury in rats via regulation of HMGB1/TLR4/NF-kB signaling pathway. Cell Physiol Biochem 43: 481-491, 2017.

7. Ding H, Jiang L, Xu J, Bai F, Zhou Y, Yuan Q, Luo J, Zen K and Yang J: Inhibiting aerobic glycolysis suppresses renal interstitial fibroblast activation and renal fibrosis. Am J Physiol Renal Physiol 313: F561-F575, 2017.

8. Andújar I, Ríos JL, Giner RM and Recio MC: Pharmacological properties of shikonin-a review of literature since 2002. Planta Med 79: 1685-1697, 2013.

9. Yang Y, Wang J, Yang Q, Wu S, Yang Z, Zhu H, Zheng M, Liu W, $\mathrm{WuW}, \mathrm{He}$ J,etal:Shikonin inhibits the lipopolysaccharide-induced release of HMGB1 in RAW264.7 cells via IFN and NF- $\kappa B$ signaling pathways. Int Immunopharmacol 19: 81-87, 2014.

10. Wang SY, Yang XJ, Yang SS, Wang W, Tian YY, Cao FL and Zhou J: Association analysis of cytokine polymorphisms and plasma level in Northern Chinese Han patients with paroxysmal nocturnal hemoglobinuria. Chin Med J (Engl) 125: 1576-1580, 2012.

11. Chen Y, Zheng L, Liu J, Zhou Z, Cao X, Lv X and Chen F: Shikonin inhibits prostate cancer cells metastasis by reducing matrix metalloproteinase-2/-9 expression via AKT/mTOR and ROS/ERK1/2 pathways. Int Immunopharmacol 21: 447-455, 2014.

12. Ning L, Ma H, Jiang Z, Chen L, Li L, Chen Q and Qi H: Curcumol suppresses breast cancer cell metastasis by inhibiting MMP-9 via JNK1/2 and Akt-dependent NF- $\kappa$ B signaling pathways. Integr Cancer Ther 15: 216-225, 2016.

13. Scherberich A, Tucker RP, Degen M, Brown-Luedi M, Andres AC and Chiquet-Ehrismann R: Tenascin-W is found in malignant mammary tumors, promotes alpha8 integrin-dependent motility and requires p38MAPK activity for BMP-2 and TNF-alpha induced expression in vitro. Oncogene 24: 1525-1532, 2005. 
14. Su Y,Huang N, Chen D, Zhang L, Dong X, Sun Y,Zhu X, Zhang F, Gao J, Wang Y, et al: Successful in vivo hyperthermal therapy toward breast cancer by Chinese medicine shikonin-loaded thermosensitive micelle. Int J Nanomedicine 12: 4019-4035, 2017.

15. Jang SY, Lee JK, Jang EH, Jeong SY and Kim JH: Shikonin blocks migration and invasion of human breast cancer cells through inhibition of matrix metalloproteinase-9 activation Oncol Rep 31: 2827-2833, 2014.

16. Jiang W, Tian W, Ijaz M and Wang F: Inhibition of EGF-induced migration and invasion by sulfated polysaccharide of Sepiella maindroni ink via the suppression of EGFR/Akt/p38 MAPK/MMP-2 signaling pathway in KB cells. Biomed Pharmacother 95: 95-102, 2017.

17. Thakur R, Trivedi R, Rastogi N, Singh M and Mishra DP Inhibition of STAT3, FAK and Src mediated signaling reduces cancer stem cell load, tumorigenic potential and metastasis in breast cancer. Sci Rep 5: 10194, 2015.

18. Nie Y, Yang Y, Zhang J, Cai G, Chang Y, Chai G and Guo C: Shikonin suppresses pulmonary fibroblasts proliferation and activation by regulating Akt and p38 MAPK signaling pathways. Biomed Pharmacother 95: 1119-1128, 2017.

19. Takaku S, Shimizu M and Takahashi H: Japanese Kampo medicine ninjin'yoeito synergistically enhances tumor vaccine effects mediated by CD8 ${ }^{+} \mathrm{T}$ cells. Oncol Lett 13: 3471-3478, 2017.

20. Borhani S, Mozdarani H, Babalui S, Bakhshandeh M and Nosrati H: In vitro radiosensitizing effects of temozolomide on U87MG cell lines of human glioblastoma multiforme. Iran J Med Sci 42: 258-265, 2017.

21. Hsu PC, Huang YT, Tsai ML, Wang YJ, Lin JK and Pan MH: Induction of apoptosis by shikonin through coordinative modulation of the Bcl-2 family, p27, and p53, release of cytochrome c and sequential activation of caspases in human colorectal carcinoma cells. J Agric Food Chem 52: 6330-6337, 2004.

22. Nam KN, Son MS, Park JH and Lee EH: Shikonins attenuate microglial inflammatory responses by inhibition of ERK, Akt, and NF-kappaB: Neuroprotective implications. Neuropharmacology 55: 819-825, 2008.

23. Baradaran PC, Mohammadi A, Mansoori B, Baradaran SC and Baradaran B: Growth inhibitory effect of Scrophularia oxysepala extract on mouse mammary carcinoma 4T1 cells in vitro and in vivo systems. Biomed Pharmacother 85: 718-724, 2017.

24. Gong K and Li W: Shikonin, a Chinese plant-derived naphthoquinone, induces apoptosis in hepatocellular carcinoma cells through reactive oxygen species: A potential new treatment for hepatocellular carcinoma. Free Radic Biol Med 51: 2259-2271, 2011.
25. Mao X, Yu CR, Li WH and Li WX: Induction of apoptosis by shikonin through a ROS/JNK-mediated process in Bcr/Abl-positive chronic myelogenous leukemia (CML) cells. Cell Res 18: 879-888, 2008

26. Liu T, Xia Y, Li J, Li S, Feng J, Wu L, Zhang R, Xu S, Cheng K, Zhou Y, et al: Shikonin attenuates concanavalin A-induced acute liver injury in mice via inhibition of the JNK pathway. Mediators Inflamm 2016: 2748367, 2016.

27. Kim KY, Park KI, Kim SH, Yu SN, Park SG, Kim YW, Seo YK, Ma JY and Ahn SC: Inhibition of autophagy promotes salinomycin-induced apoptosis via reactive oxygen species-Mediated PI3K/AKT/mTOR and ERK/p38 MAPK-dependent signaling in human prostate cancer cells. Int J Mol Sci 18: 1088, 2017.

28. Yoshida K, Kuwano K, Hagimoto N, Watanabe K, Matsuba T, Fujita M, Inoshima I and Hara N: MAP kinase activation and apoptosis in lung tissues from patients with idiopathic pulmonary fibrosis. J Pathol 198: 388-396, 2002.

29. Khalil N, Xu YD, O'Connor R and Duronio V: Proliferation of pulmonary interstitial fibroblasts is mediated by transforming growth factor-beta1-induced release of extracellular fibroblast growth factor-2 and phosphorylation of p38 MAPK and JNK. J Biol Chem 280: 43000-43009, 2005.

30. Ahn J, Won M, Choi JH, Kim YS, Jung CR, Im DS, Kyun ML Lee K, Song KB and Chung KS: Reactive oxygen species-mediated activation of the Akt/ASK1/p38 signaling cascade and p21(Cip1) downregulation are required for shikonin-induced apoptosis. Apoptosis 18: 870-881, 2013.

31. Shahsavari Z, Karami-Tehrani F and Salami S: Shikonin induced necroptosis via reactive oxygen species in the T-47D breast cancer cell line. Asian Pac J Cancer Prev 16: 7261-7266, 2015.

32. Lan W, Wan S, Gu W, Wang H and Zhou S: Mechanisms behind the inhibition of lung adenocarcinoma cell by shikonin. Cell Biochem Biophys 70: 1459-1467, 2014.

33. Nguyen QD, Challapalli A, Smith G, Fortt R and Aboagye EO: Imaging apoptosis with positron emission tomography: 'bench to bedside' development of the caspase-3/7 specific radiotracer [(18) F]ICMT-11. Eur J Cancer 48: 432-440, 2012.

34. Hong D, Jang SY, Jang EH, Jung B, Cho IH, Park MJ, Jeong SY and Kim JH: Shikonin as an inhibitor of the LPS-induced epithelial-to-mesenchymal transition in human breast cancer cells. Int J Mol Med 36: 1601-1606, 2015. 